\title{
Learning Chinese Mandarin characters in an English-speaking country: The development of a child's symbolic mind
}

\author{
Wenjie Wang', Annabelle Black Delfin ${ }^{2}$
}

\begin{abstract}
This qualitative research explores the development of the symbolic mind in children through learning Chinese Mandarin characters. Navigated through the lens of relational developmental system metatheory and guided by Vygotsky's sociocultural theory, findings present the analysis of the developmental processes in children's recognition of symbols and use of known symbols to make and share meaning. This study also offers an explanation of the effect of changes in the sociocultural environment on children's symbolic development. Further, cultural differences toward symbolic representation are discussed with the recommendation of focusing on recognition followed by writing when learning Chinese Mandarin characters.
\end{abstract}

\author{
Article History \\ Received: 28 November 2020 \\ Accepted: 15 March 2021

\section{Keywords} \\ Symbolic development; \\ Symbolic representation; \\ Early childhood; Chinese \\ Mandarin characters; \\ Relational developmental \\ systems metatheory; \\ Sociocultural theory
}

\section{Introduction}

Symbol systems have existed and have been utilized for communication throughout history (Vallotton and Ayoub, 2010). Symbols, symbolic play, symbolic thought, symbolic development, symbolic interpretation, and symbolic representation have been widely studied across multiple fields (DeLoache, 2004; Uttal and Yuan, 2014; Wu, 2013). Symbolic representation is considered an important phenomenon in children's cognitive development and language learning (Smith and Jones, 2011). For our purposes here, symbolic representation refers to developmentally dependent abilities to discern symbolic meaning, to interpret symbolic meaning, and to use symbols to convey or communicate meaning. However, on the one hand, most studies underline symbolic representation for learning an alphabetic language, but less research attention is given to non-alphabetic languages such as Chinese Mandarin ${ }^{1}$, which is characterized by its non-alphabetic orthography. On the other hand, learning Chinese Mandarin is becoming more and more popular in the United States. Numerous research studies have been conducted primarily focused on teaching Chinese Mandarin as a second language to a non-Chinese population, teaching Chinese Mandarin as a heritage, and developing Chinese Mandarin programs and curricula for populations of Chinese descent (Wu, 2013). Chinese Mandarin has been studied from listening, speaking, reading, and writing perspectives with specific investigations on mastering Chinese Mandarin characters among youth, but there is less research exploring how young children learn Chinese characters from the lens of symbolic development, especially to those who are temporarily residing in the United States.

With such background that is described above, we were very interested in comprehending how Chinese children develop cognitive abilities to perceive meaning from symbols in the course of learning Chinese Mandarin characters. This is of particular interest, from a developmental perspective, when taking into consideration that the preschool children in this study have been learning characters when residing in

\footnotetext{
${ }^{1}$ New Mexico State University, Glass Family Research Institute, Las Cruces, NM, USA, e-mail: wenjie@nmsu.edu, ORCID: https://orcid.org/0000-0002-3103-8445

${ }^{2}$ New Mexico State University, Curriculum and Instruction, Las Cruces, NM, USA, e-mail: kablack1@nmsu.edu, ORCID: https://orcid.org/0000-0001-6343-5528
}

${ }^{1}$ For the purposes of this article, unless otherwise specified, the words "Chinese" and 'character/s' refer to Chinese Mandarin (simplified) characters. 
the United States, and not immersed in Chinese culture. The research inquiry for this study is about children's development in understanding and using symbolic representation in the process of learning characters. In attempting to acquire some understanding of these developmental processes, the research questions for the study are:

1) How a child's symbolic mind is developed when learning Chinese Mandarin characters?

2) How do Chinese children learn to discern meaning from the symbols represented in Chinese characters?

3) How do cultural and social environments influence children's learning of Chinese Mandarin characters when they are temporarily residing in the United States?

This study aims to contribute to the educational field, especially in the area of early childhood with a focus on symbolic representation in learning non-alphabetic languages. Parents, teachers, early caregivers, school administrators, and other educators are able to refer to the collected information on symbols, symbolic understanding, visual object recognition, symbolic function, symbolic play, symbolic representation, and concept acquisition in children's learning and teaching. Much of this work is cited here, comprising many underpinning features that make up the abilities we refer to as symbolic representation. This paper incorporates previous work that examines the understanding and use of symbols and has applied that to a non-alphabetic language system, also taking into account what experiences Chinese families have had, especially in improving learning Chinese characters with a change of social and cultural environment. Besides, the cases in this study may serve as examples to families of how to advance nonalphabetic language learning, such as Chinese characters, and this study may call attention to how symbolic representation and concept acquisition works in children's cognitive development from the very beginning with appropriate strategies. In addition, teachers, educators, and researchers may benefit by having more detailed information about the role of symbolic representation in the Chinese language and the nonalphabetic system.

\section{Theoretical Framework}

In seeking to better understand developmental processes in young children, this research is focused on some of the most uniquely human capacities; symbolic representation and language. Examining these topics through a developmental framework, it is important to note that, while at times, we may refer to cognitive or linguistic development, our developmental lens is not ordered in distinct stages or learning domains. Traditionally developmental theories have conceptualized child development as a teleological and, mostly, linear movement toward increasing complexity. Child development is often portrayed as progressions in mental structure and successively higher skill level, whereby each successive 'level' encompasses previous 'levels', allowing for increasing mental complexity and skill acquisition (Taylor, 2016). While we do agree that development typically proceeds toward greater complexity, we do not see it progressing as parallel lines of ages, stages, and learning domains that never meet. Cognitive development encompasses, among other things, symbolic representational abilities, abstract thinking and language learning. These three aspects of cognitive development are part of a relational system that produces cognitive processes such as comprehension, conveyance, and communication.

As such, this line of inquiry comes through the lens of relational developmental systems metatheory (Overton, 2013). Relational developmental systems offer a holistic, context-based perspective on developmental sciences. In relational developmental systems, development is seen as emergent from the relations of interacting aspects of the whole system. Overton (2013) explains, "It is through complex reciprocal bidirectional and circular reciprocal interpenetrating actions among the co-acting parts that the system moves to levels of increasingly organized complexity" (p. 53). The system in this case would refer, not only to the child, but also to the environment in which the child is contextualized and situated. Relational developmental system metatheory is not another Cartesian developmental theory pitting the influence of a discrete 'environment' against the influence of a predetermined biology. Instead the child's development is understood to emerge from the relations of the system's interdependent parts; ancestry, child, family, culture, community, society, language, and others. Development, then, is an emergent 
quality of an adaptive system, where the parts are unique to each child, place, and time, thus making the behavior of the whole system undetermined (Overton, 2013). A main principle of relational developmental systems metatheory is that people have agency and have a significant impact on their environment, bodies, and development. Individual considerations including, "biological, demographic, personality, cognitive, motivational, emotional, and behavioral characteristics, in conjunction with context-specific variables explain variation or consistency in developmental trajectories over time" (Gayman, Fraser-Thomas and Baker, 2017).

With this holistic view on children's development, it was impossible to explore development, especially focusing on factors as centrally situated as symbols and language, without deeply considering the culture and society in which a child is embedded when developing the capacity for symbolic representation. Hence, we have also employed Vygotsky's (1978) sociocultural theory as a theoretical framework through which to analyze this research. Vygotsky offers several metaphorical devices and routes to understanding the effect/s of language and culture on development. The sociocultural theory has been widely used in various studies regarding language teaching and learning, based on the belief that the process of language learning is always being socially constructed and reconstructed (Menezes, 2013) with cultural tools (Vygotsky, 1978). On the one hand, tools, in Vygotsky's (1978) understanding, exist in two categories, technical tools such as texts, books, materials, media, digital devices, and psychological tools such as language, signs, and symbols. Both play crucial roles in children's cognitive development and concept acquisition. On the other hand, Vygotsky (1978) views language as not only a technical tool for communication, but also a psychological tool that promotes cognitive development through constructing meaning between humans and their linguistic objectives (Hammond, 2002). Therefore, children's language learning involves both types of tools through social interactions. Children convert/transform from technical tools to their own representations, symbols, patterns, and meaning when they have conversations and other types of communications in the context of social interactions, and in turn, social interactions internalize children's understanding of the language, form the individual's perceptions toward language practices, and establishes foundations for further language progress and growth (Gee, 1990; Vygotsky, 1978).

In this research study, sociocultural theory informs us that children learn Chinese characters through interaction with technical tools such as books and apps, as well as through social and linguistic interactions with others. Children became symbol-minded through deconstructing characters from abstract concepts to concrete visuals and reconstructing them with meaning-making by acknowledging the engagement of the learning environment such as family communication, school collaboration, peer influence, and community participation. With this being said, sociocultural theory and relational developmental systems metatheory guide us to a deeper exploration of the relationship between two main aspects of cognitive development; the symbolic mind and language learning, as well as a broader understanding of children's concept acquisition and symbolically relevant mental activities and deeds.

\section{Review of the Literature}

Based on the background of the study and focused on the research questions, this study provides reviews from two paths, 1) the development of cognitive capacities that involve symbolic representation and language learning and 2) learning Chinese Mandarin characters.

\section{Children's Symbolic Representation in Language Learning}

As this paper seeks to provide some explanation for the developmental processes that account for meaning-making from symbols, especially when learning language, it proved useful to examine the literature that has deconstructed the levels of complexity in representational abilities. We begin by examining symbols and early symbolic understanding in young children. As children grow they begin to incorporate visual object recognition, object substitution in play and gain understanding of the symbolic function. Further cognitive development leads most children to the ability to discern, convey and communicate meaning from and through symbols; symbolic representation, which underpins deeper 
understanding and concept acquisition. These developmental progressions in cognitive complexity have been studied by many in the field. Here we present a brief overview of children's symbolic representation in language learning.

In discussing children's cognitive development, including symbolic representation and language learning, Piaget and Bruner's theories must be first highlighted as they are pioneers who studied symbols in children's development. Inhelder and Piaget (1964) advocated that children should be able to make connections and find relations among abstract concepts, as well as have abstract thinking ability when they learn to read written language. Piaget further suggests that until children are about 12 years old, they do not fully acquire these abilities because children need to first develop symbolic and operational thinking abilities (Piaget, 1950). In a similar vein, Bruner (1966) also said that there are three modes of representation relating to children's development, they are, enactive, iconic and symbolic, and symbolic is the foundation for children's language learning. Both Piaget and Bruner believed that symbols are keys to support children's symbolic development which further advances children's cognitive development including abstract thinking skills and symbolic representational abilities. This can be seen, especially in areas of language learning, such as when children express themselves through mark making (Abbott, 2000; Pampoulou and Detheridge, 2007). Written expression is evidence of a child's relationship with symbols (Athey, 2007).

Symbol and symbolic understanding. A symbol can be any number of things as long as it is intended to represent something else and can be interpreted that way by another person. Uttal and Yuan (2014) state that, "to use a symbol, children need to 1) understand the intention that led to the creation and use of the symbol and 2) how the symbol relates to its referent" (p. 295). Bruner (1966) also asserted that objects include social objects or people, physical objects or things, and abstract objects or ideas (p. 10). In language learning, Vygotsky $(1981 ; 1987)$ proposed that symbols are also tools for serving social-emotionalcognitive functions, namely, communication and representation. As such, Uttal and Yuan (2014) explain that there are three components of symbolic understanding: a symbol, its referent, and the human interpretation that discerns the relationship between the symbol with its referent. The intention of both the creator or user of the symbol and the interpreter or receiver of the symbol is central to the final meaning that can be taken from the symbolic representation.

Several research studies have shown that children as young as three years old use symbols to interact with the world and then use language/words to communicate with others as well as to represent more complicated social concepts (DeLoache, 2004; Namy and Waxman, 2005; Vallotton and Ayoub, 2010). A child may not know how to read or write but can understand the intention of using spoken sounds to represent words; comprehension of words constitutes a form of symbolic understanding (Uttal and Yuan, 2014). DeLoache (2004) has explored young children's use and creation of symbols. She showed how children's understanding of intention is evident in symbol production. A study was conducted where children were asked to make a drawing to convey information to an adult about a game. When the children were told that more information was needed on the drawing to make sense to the adult, the children added more marks on the drawing to provide more information. This research demonstrated that the children understood the communicative aspect of the drawings; i.e. that the drawing's function was to convey information (DeLoache, 2004). This research further demonstrated that children's ability to understand the communicative function and shared meanings of symbolic representation coincides with the age at which many developmental theorists believe children develop the cognitive functions called Theory of Mind, or the ability to perceive emotional and intellectual states in others (Mercer, 2018).

Visual object recognition and symbolic function. Children's visual object recognition and play with objects (symbols) can be related to children's early language development because children use various objects and substitute one object for another to interact/communicate (Smith and Jones, 2011). The ability to employ object substitution in play or communication depends on sufficient development in visual object recognition. This refers to a child's ability to recognize visual objects, even when they are represented in such a sparse and geometric way as to be a symbol of the visual object. With this being said, according to Smith and Jones (2011), "the tie between object substitutions and language development is classically 
attributed to a shared 'symbolic function'" (p. 2). They propose that a symbol-like object is tended to be substituted by younger children in implementing the symbolic function.

Symbolic play and symbolic thinking. With the understanding of object substitutions and symbolic function, symbolic play is introduced and extended to a broader umbrella with "a range of pretend play behaviors including dress-up and role-playing" (Smith and Jones, 2011, p. 1). Starting from here, the ability to use a symbol to represent something else is often referred to as symbolic development which is "a set of abilities that enable humans to represent concepts in their absence" (Vallotton and Ayoub, 2010, p. 2). Symbolic thinking develops from children's increasing understanding of the symbolic function and encounters with symbols in the course of interacting with others. Specifically, it refers to children's ability to understand concrete concepts, as well as abstractions, and from initially a physical standpoint to both physical and mental stances (Vallotton and Ayoub, 2010).

Symbolic development and symbolic representation. Clarifying the aforementioned terms is significant for understanding how children make further progress in symbolic development. Vygotsky (1987), Piaget and Inhelder (1969) viewed children's symbolic play as a crucial stage in developing children's symbolic representation. According to Veraksa and Veraksa (2016), "symbolic representation is understood as a special form of mental representation of a learned object or a phenomenon in the mind where the latter are represented through their external (as opposed to their content) traits that can be substituted by some other object or a phenomenon" (p. 669). In play, children have opportunities to develop their capacity for concept formation and symbolic representation through "use of gestures, speech, and written signs in an imaginary situation" (Wu, 2013, p. 13) and have chances to operate and practice the nature of social rules that both contribute to children's language development. An example of this can be seen when a child pretends something like a deck of playing cards is a cell phone and has a pretend conversation. Black Delfin (2020) demonstrated how everyday play objects can become infused with symbolic meaning in pretend play and used by children to construct and define aspects of their own identities and their understandings of discursive information that surrounds them. Vygotsky (1987) also agreed with the importance of symbolic representation, and he viewed that symbolic systems or signs, as used by humans could be used to, not only, change the environment, but also to transform and master themselves.

Concept acquisition. In short, younger children under three years old mainly use symbols-objects to engage with the world and then these symbols-objects are gradually replaced by symbolic representation in the form of language/words. In this process, visual object recognition, object substitution, and play with objects are crucial signals to assess children's language development (Smith and Jones, 2011). Although the symbolic function is implemented at a superficial level by younger children, symbolic development is further deepened as they grow up. This deepening of symbolic development results in concept acquisition. As children have more experiences and social/ linguistic exposure to symbols and symbolic thought, they begin to arrange the knowledge gained into broader concepts that include symbols. Vygotsky (1987) described two types of concept acquisition; 'every day' and scientific concepts. Every day conceptual understanding arises from the day-to-day experiences of children's lives. In this way, everyday concepts exist in the sensory and concrete, as this is where most young children are developmentally in the early years. Everyday concepts serve as a foundational understanding of the material world and prepare the child for comprehending scientific concepts. Fleer and Raban (2006) describe Vygotsky's concept acquisition model with everyday concepts developing from "below (concrete) to above (abstract)... and scientific concepts develop from above to below, from the more complex to the more elementary" (p. 70). This two-way avenue of concrete to abstract/ abstract to concrete was noted by Vygotsky, "the weakness of the everyday concept lies in its incapacity for abstraction. In contrast the weakness of the scientific concept lies in its insufficient saturation of the concrete" (1987, p 169). Vygotsky suggested that the two types of concept acquisition- every day (concrete) and scientific (abstract) merge together in a mutually reinforcing relationship that allows deep thought to take place. 


\section{Learning Chinese Mandarin Characters}

As a print system that is non-alphabetic, in Chinese the concept of what makes up a word is structured differently than alphabetic orthographies where words are composed of letters that have an auditory connection to the written symbol or letter. Chinese word learning involves the radical, the character, and the word.

Radicals are individual units, made of strokes. Chinese characters are made from radicals, usually two or more. Radicals can indicate meaning in a character (semantic) or indicate phonetic clusters that cue pronunciation similar to rime in alphabetic systems (Yeh, Chou and Ho, 2017). For example, the water radical (沫 ) can be found in the characters lake (湖), ocean (海), and river (河). In this way, words in Chinese can be comprised of one or more characters, making word definition in print more difficult to isolate compared to alphabetic print systems where there is a designated auditory sound for each letter, with each sound mapped onto a letter and a word is formed. According to McBride (2016), "Chinese characters represent syllable-level sounds" (p. 526). However, Chinese writing does not directly reflect representations of speech sounds, rather "one Chinese character maps onto a single syllable which is usually also a single morpheme or meaning unit" (McBride, 2016, p. 527). As many radicals are phonetic and indicate the pronunciation of the character, Tong, Tong and McBride (2017) point out that young children gain access to character configuration knowledge visually, followed by auditory cues to pronunciation. They state, "using radical cues in encoding novel Chinese characters is a gradual developmental process" (p. 1263). Their research implies that young children's first access to understanding meaning of characters relies on sensory pathways (vision and hearing) that develop before neural abilities for processing of abstract concepts.

There are about 2500 to 5000 commonly used Chinese characters that should be memorized which sounds impossible, but they are introduced with six different categorizations. They are, 1) pictographs; 2) explicit characters; 3 ) associative compounds; 4 ) pathopoeic characters; 5) mutually explanatory characters; and 6) phonetic loan characters $(\mathrm{Wu}, 2016$; Sun, 2015).

Pictographs represent the shape of certain objects or their parts such as 木 (mù, wood; tree), 刀 (dāo, knife), 女 (nŭ, woman). Explicit characters are simple diagrammatic indications of abstract ideas such as 上 (shàng, above), or下 (xià, below). Associative compound characters are derived from their components, which may combine two or more ideographs such as, 明 (míng, bright, the combination of 日 rì, sun and 月 yuè, moon), and 森 (sēn, forest, the combination of three trees 木 mù). The majority of Chinese characters are pictophonetic, which combines semantic and phonetic components. For instance, the character 妈 (māa, mother) consists of 女 (nü, female) and 马 (mă, horse); 女 suggests the general meaning of the character while 马 signals its pronunciation. Mutually explanatory (or synonymous) characters refer to those that are of the same or similar meanings and thus can be used to define one another $(\mathrm{Wu}, 2016)$. Phonetic loan characters refer to those that originally had no written form and so borrowed existing characters of the same or similar pronunciation. For example, the character 我 (wǒ) resembles a weapon with a saw-toothed blade and long shaft, and originally referred to a kind of ancient weapon. Because the pronunciation of this character is like that of the pronoun "I", 我 was borrowed to mean "I" or "me" (Sun, 2015).

With the above descriptions of Chinese characters, in analyzing how character recognition is taught in China (the mainland), Hong Kong, and Taiwan, McBride (2016) emphasizes that character learning usually relies on strong visual-spatial skills to discriminate between the subtle differences in how the characters are written or printed. This is combined with repetition in recreating or copying the characters, resulting in visuo-motor long-term muscle memory. Repeated encounters with Chinese characters afford memorization. It is also worth noting that simplified Chinese characters are taught and used in China mainland, while traditional Chinese characters are applied in Hong Kong and Taiwan. The differences between simplified Chinese characters and traditional Chinese characters is the former uses fewer strokes for complex characters while the latter maintains the same. Thus, simplified Chinese characters are easier and faster to write compared to traditional Chinese, which is more visually complex. 
In thinking about how children discern meaning from character recognition, one might ask what is the difference between memorization and recognition? What is recognition, if not to identify a visual memory? Do children discern meaning from symbols because an ascribed meaning has discursively been assigned and thus, the meaning associated with that symbol has been 'learned' or memorized to later be 'recognized'? It would seem that meaning is, at first, 'learned' and later recognition is applied (i.e. the memory of the assigned meaning is recalled and applied to the symbol.) With memorization and recognition central to Chinese character learning, over time proficient Chinese learners recognize characters in somewhat the same way that proficient English learners can recognize words as 'sight words', where the character or word is not decoded but the meaning is memorized and recognized in whole (McBride, 2016).

Researchers Lu, Wu, Fadjo \& Black (2010) have more recently proposed integrating bodily movement and response in learning Chinese characters. Their work, along with others (Fadjo, Lu and Black, 2009; Glenberg, 2008) in the area of instructional embodiment is grounded in theories of embodied cognition. Embodied cognition (Gibbs, 2006), like relational developmental systems, does not recognize a mind/body split; instead cognition is understood to be constituted from the lived experience in a human body that interacts with the physical and cultural environments. Cognition, then, is seen as emergent from the body's interaction with others and in the environment in which it is embedded. Instructional embodiment is grounded in the idea that the mind is embodied, and as such, instruction or learning is more comprehensive if the body is involved in the process of taking up the new instructional content, in this case learning characters. Lu et al. (2010) explain, "The kinesthetic action of a person has its importance on how he or she thinks, perceives, learns, uses language, and experiences consciousness, feelings, and the world" (p. 2487). Instructional embodiment in the case of Chinese character learning could involve learning to write characters and incorporating characters into drawings. Additionally, many of the forms of instructional embodiment available involve computer technologies that facilitate the embodied experience; for example, a student wearing a webcam while writing Chinese characters and, hence also seeing themselves move (their hands) on the computer screen as their hands form the characters. This perceptual feedback loop serves to amplify the already existing interaction of body, space, materials, content, and cognition that situates the symbolic understanding within the body (Lu et al., 2010).

\section{Research Design and Method}

Using a qualitative case study approach helped both the authors and the participants of the study to recognize multiple perspectives of the research as well as provided a more in-depth understanding of the research questions. This research study was designed as a two-case study. Purposeful sampling was used in order to focus on Chinese families who temporarily live in the United States and have China-born parents and China-born children who are bilingual learners between four to five years old.

\section{Research Context and Participants}

Families were selected from a local university at City X (pseudonym) which is located in southern New Mexico. Although the White and Hispanic/Latino are the majority population, a range of diversity is represented by the local university. With this wide range of diversity, we initially planned to conduct the research face to face at the university campus, however, a state lockdown occurred due to Covid-19 and we had to modify our research plan. After seeking suggestions from the Institutional Review Board (IRB), the entire research changed from person-to-person to online, that is, call for participants, recruitment of participants, purposeful sampling, consent process, data collection and member check were all processed through emails and online meetings. First, an email of call for participants was sent through our personal and professional contacts. In specific, the call for participants invited people who are, 1) a Chinese-born parent; 2) a temporary resident in the United States and 3) teaching their own child/children Chinese characters to participate in this study. To those people who thought they were qualified and responded to this email, we then sent a recruitment email with a detailed explanation of the research study such as the research questions, benefits and risks, rights and confidentiality with options of scheduling a phone call for clarifying questions if they need. We also stressed that if they decided to participate in this study, they 
are free to withdraw from the research study at anytime and anywhere without any penalty.

In other words, purposeful sampling was used in recruitment of participants. According to Patton (2002), purposeful sampling is an effective strategy in qualitative research for identifying and selecting cases with limited resources. Cresswell and Plano Clark (2011) also address that purposeful sampling involves classifying and determining a group of people who experience and share a common phenomenon or interest. With purposeful sampling, a consent form was sent to potential participants to complete, sign and return to us. As a result, two families, family A and family B were selected. There were a total of five participants, two mothers and three children. Family A is Flora and her daughter, Kayla. Family B is Zara and her twin sons, Aden and Alex. All of them are China-born but temporarily living in the United States. Both mothers are language teachers who were bilingual in Chinese Mandarin and English and paid great attention to language teaching and learning to their child/children. All of the children are bilingual (English and Chinese Mandarin) learners in both countries and they were from four to five years old. Detailed information is provided in the following table.

Table 1. Participant Information

\begin{tabular}{ccccc}
\hline Case/Family & Participants & Age & Gender & Length of stay in the U.S. \\
\hline & Flora & 42 & Female & 1 year \\
\cline { 2 - 4 } & Kayla & 5 & Female & 1 year \\
\hline B & Zara & 44 & Female & 2 years \\
\cline { 2 - 4 } & Aden & 4 & Male & Half-year \\
\hline
\end{tabular}

\section{Methods of Data Collection}

We used a parent questionnaire (see Appendix A), parent interview, and children's products such as children's writings and drawings as data. The three methods provided us with rich and first-hand information that built a foundation for analysis. In order to further process information in a way that gives both breadth and depth, parents were first invited to complete a parent questionnaire via email. Following receipt of the parent questionnaire, interviews were then scheduled and conducted through online meetings for further clarification and comprehension. All interviews were recorded and transcribed, and the interview questions (see Appendix B) were semi-structured. Depending on the participants' responses, follow-up questions were also added. In terms of children's products such as drawings and writings, Thompson (1995) says that children's products are one kind of picture language which represents children's personal expression and intrinsic thoughts. Hence, as important evidence of symbolic development, children's drawings and writings demonstrate their thinking and learning, namely, cognitive development (Thompson, 1995). These were also collected for data interpretation. Depending on participants' selections, participants scanned their child/children's drawings and writings, shared with us via email and talked about them through online interviews.

\section{Methods of Data Analysis}

Qualitative content analysis and visual analysis were used to interpret the data. These two methods helped us to carefully explore the role of symbolic representation in Chinese character learning. On the one hand, qualitative content analysis was applied to analyze the parent questionnaire and interview data via an inductive approach by Saldaña's (2009) The Coding Manual for Qualitative Researchers. Steps such as coding and grouping, categorization, abstraction, comparing, and generalizing were followed in this process. On the other hand, children's drawings and writings were analyzed by visual analysis. Elkins (2003) states that in order to help researchers to navigate data, explore relationships, and understand complex concepts, visual data analysis is a way of discovering and comprehending patterns via visual interpretation. Combined with qualitative content analysis and visual data analysis, findings were 
organized and reported together.

\section{Findings and Analysis}

Findings are constructed and analyzed by responding to the research questions one by one. In responding to the first question of how Chinese children learn to discern meaning from the symbols represented in Chinese characters, the analysis focused on switching between a concrete referent and a symbol. In discussing the second question of how a child's symbolic mind is developed when learning characters, analyses mainly include: 1) applying known symbols to reproduce more meaning, and 2) using symbols and simple storytelling to share meaning from daily experiences. In explaining the third question of how changes in cultural and social environments influence children's Chinese character learning when they are temporarily residing in the United States, the main theme is interpreted as increased interactions with technical tools and decreased interactions with the community.

\section{How do Chinese Children learn to Discern Meaning from the Symbols Represented in Chinese Characters?}

\section{Switching between a Concrete Referent and a Symbol}

Both families highlighted digital tools that helped children understand Chinese characters. Both families used an app, named Wukong Literacy (2020) which is featured by its animation demonstration of the transformation of Chinese characters. As discussed earlier in the literature review, Chinese characters are symbols and they originally come from the actual things, in other words, how the actual things, or referents, slowly transform into the characters. Throughout history, Chinese characters get reformed, changed, and simplified and this accounts for why the current characters look different from the earlier days. Table 2 includes a few examples-the screenshots from the app that displays the transformation from referents to symbols by animation demonstrations when children learn characters.

Table 2. Examples of character transformation from a concrete referent and a symbol (Wukong Literacy, 2020)

Actual thing Transformation

As the animations demonstrate the transformation of Chinese characters, children can see how the character describes a concrete referent and over time became a symbol for the referent. However, actually learning to recognize characters requires that learners can mentally construct and deconstruct the symbol from its concrete origin to its symbolic representation. As young children experience and learn about reality, the understanding of the material, or concrete, world occurs first in their development. With time, 
children begin to acquire the capacity for abstract understanding. van Oers and Poland (2007, p. 14) state that abstraction is 'a dialectical process between the concretely given objects and the abstract representations of them' where symbolic representations are constructed as mental objects, which sustain 'inner relationship' with the concrete world" (van Oers, 2001, p. 287). This 'inner relationship with the concrete world' is reflective of the directional sequence of a child's lived experience with concrete encounters preceding and providing a foundation for neural development that enables abstraction.

Because of this, reality is usually constructed by young children hierarchically from a concrete understanding of material objects to more subtle abstractions of the object/s that evolve into symbolic representation, or in this case Chinese characters. The development of the ability to discern meaning from symbolic representation is linked with the child's progressing ability to "detach from the concrete world, while maintaining connection with it through representations" (Otsuka and Jay, 2017, p. 994). Once the ability to conceptually hold both the concrete and the symbolic is achieved, then children can conversely begin to understand abstractions or symbols by deconstructing their meaning back to the concrete origin.

This ability to comprehend the concrete/abstract relationship of symbols through construction and deconstruction allows children to discern meaning from symbols, recognize new symbols, and to learn characters. Specifically, since the level of character, learning speed and total time spent working with the app can be set up by parents, both families in this study only required their children to learn five new characters per day within 30 minutes due to children's age and their capability of learning. Children comprehended new characters from a concrete referent to abstract through watching character transformation, namely, animation demonstration. Based on each individual's development and progress, parents can add a section of character writing when children's fine motor skills are improved. When the section of character writing is added, children can move from character recognition to character writing through practicing character stroke order that the app offers. Thus, working with an app, such as described above that visually demonstrates this two-way evolution of meaning, improves children's symbolic thinking skills, enhances their symbolic representation ability, and extends their imagination. Participants reflected that children enjoyed watching the animation demonstration and their recognition and memorization of characters became stronger and firmer compared to without it.

\section{How a Child's Symbolic Mind is Developed When Learning Chinese Mandarin Characters?}

\section{Applying Known Symbols to Reproduce More Meaning}

As discussed earlier, one of the main components of a Chinese character is the radical which can be a character or an abstract form of a character. Radicals usually indicate a character's meaning or pronunciation and there are about 200 radicals that construct the Chinese writing system. In other words, one must remember 200 radicals and use these radicals as a foundation to learn more characters. Along with the characters' stroke order and stroke count, learning Chinese is challenging. However, according to Wu (2016, p. v), "most radicals, for example, are pictographs, or visual representations of objects or concepts. Given a pictograph, learners can turn the character into a vivid picture, or associate the character with a shape, color, sound, smell, feeling, emotion, movement, or action". In this research study, participants also stated that children must understand radicals and then to learn more complicated characters. In order to do so, participants explained that children first memorize radicals through switching between symbols and concrete referents which is discussed in the previous section, and after that, children interpret radicals' meaning or sounds (in this research study, children only interpret radicals' meaning) when they learn more complicated characters. In this way, children continuously expand their vocabulary and symbolic capacities in applying knowledge of radicals to new characters to form new and related meanings.

One of the participants, Zara, offered an example of how her children tried to use a known symbol to reproduce more meaning of other Chinese characters. For instance, when her children, Aden and Alex learned a character $\square$ (kǒu), means mouth, they were also explained that $\square$ (kǒu) also can be a radical in other Mandarin characters and it is possible that the radical indicates characters' meaning. Hence, when Aden and Alex saw more complicated characters which contain a radical of 口(kǒu) such as 唱, 吃, 喝, and 
吵, which all has 口 (kǒu) on the left side, they knew the meaning of the complicated characters are somehow associated with the mouth. Indeed, these characters can be viewed as verbs. The first character, 唱 (chàng) means to sing; the second character 吃 (chī) means to eat; the third character 喝 (hē) means to drink and the last character 吵 (chăo) means to quarrel and all of them are related to using the mouth. In such a way, Aden and Alex further develop their symbolic mind by applying known symbols to reproduce more meaning.

Taking 女 (nŭ) as another example. Flora shared that her child, Kayla learned a character, 女(nŭ) which means women/female, and 女(nü) is also a radical. When Kayla saw a new character, 妈(mā), she analyzed that the left part of the character is 女 (nü), which means female so the meaning of the character 妈 (mā, means mother) might be related to female. It is similar to the earlier example of $\square$ (kǒu), although Kayla didn't know the new character, she was able to guess its meaning by applying known radicals. The last example also illustrates the development of the symbolic mind. Specifically, children comprehend 水 (shui) as a character means water along with its radical I ( $^{\prime}$ is a radical which is an abstraction form of its character, 水), and they further apply its meaning of water to the following characters which contain the radical of シ such as 江(jiāng) means river; 河 (hé) means river; 湖 (hú) means lake; and 海(hăi) means ocean. Table 3 lists three examples that are discussed above.

Table 3. Examples of applying known radicals to reproduce more meaning

\begin{tabular}{|c|c|c|c|}
\hline \multicolumn{2}{|c|}{ Known symbol } & \multicolumn{2}{|c|}{ More meaning } \\
\hline Character/radical & Meaning & New character & Meaning \\
\hline \multirow{4}{*}{ 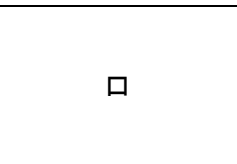 } & \multirow{4}{*}{ Mouth } & 唱 & sing \\
\hline & & 吃 & eat \\
\hline & & 掲 & drink \\
\hline & & 吵 & quarrel \\
\hline \multirow{5}{*}{ 女 } & \multirow{5}{*}{ Female } & 妈 & mother \\
\hline & & 姐 & older sister \\
\hline & & 妹 & younger sister \\
\hline & & 姨 & aunt \\
\hline & & 奶 & grandmother \\
\hline \multirow{4}{*}{ 水 $/ ;$} & \multirow{4}{*}{ Water } & 江 & river \\
\hline & & 河 & river \\
\hline & & 湖 & lake \\
\hline & & 海 & ocean \\
\hline
\end{tabular}

\section{Using Symbols and Simple Storytelling to Share Meaning from Daily Experiences}

Flora also underlined the importance of using symbols and simple storytelling while her daughter Kayla learns characters. According to Flora, using symbols and simple storytelling made Kayla relax when she learned the characters. They increased Kayla's self-motivation, which resulted in more recognizing, memorizing, and writing practice. Flora further described that Kayla enjoyed using symbols such as drawing and simple storytelling to express her ideas when she learned a new character. For example, when learning a new character of 马 (horse), it is more effective to ask Kayla to draw a horse, then write the character followed with a simple story of the drawing and text that she produced, rather than give her an order to write the character five times and remember it. In this way, Kayla's understanding of the new character's meaning is embodied (Gibbs, 2006) as she uses her bodily movements to incorporate the character (马) into a framework (in this case, drawing and writing) that employs symbolic representation to produce and convey meaning to the viewer/reader. By using symbols such as drawings and writings, and simple storytelling, Kayla not only processes symbol recognition and symbolic representation, but her memorization is also advanced.

Table 4 displays Kayla's products where Kayla used symbols and simple storytelling to share meaning from daily experiences. For example, in the first picture, Kayla drew a birthday cake with five candles because she just celebrated her five-year-old birthday. She also drew a little pig and wrote 豆 (dòu) and可 (kě) on the top of the little pig because her nickname is 可 (kě) 可 (kě) and sometimes her parents also called her豆 (dòu) 子 (zi), so together 豆 (dòu) 可(kě) is her nickname. Kayla shared her five-year-old 
celebration of blowing five candles by using a sketch of a little pig that represents herself and writing two characters that make up her nickname.

Table 4. Kayla uses symbols and simple storytelling to share meaning from daily experiences

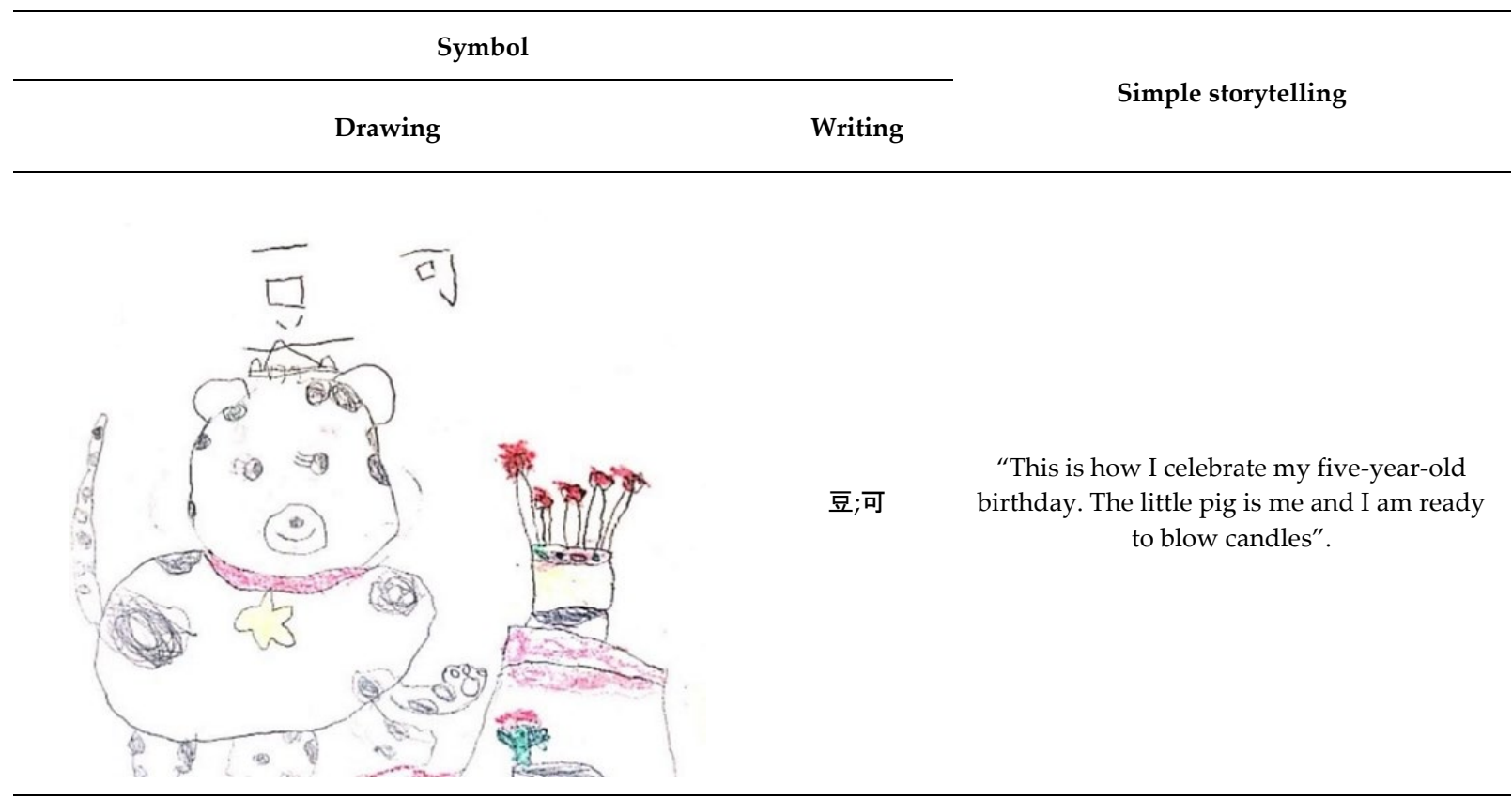
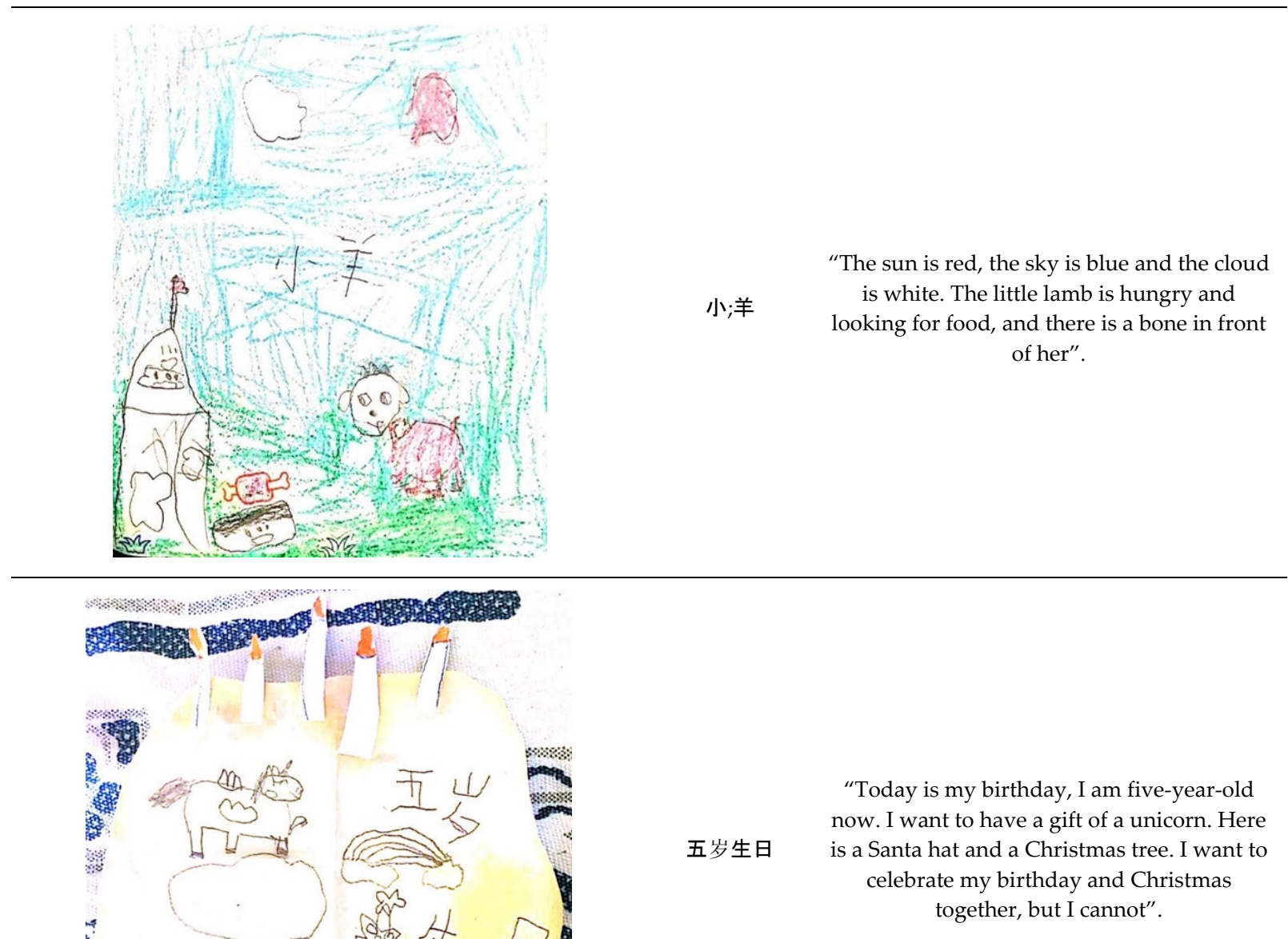

Similarly, in the second picture, Kayla drew a house, a bone and grass on the left bottom, a little 
lamb on the right bottom, and a sun with a piece of cloud on the top. She explained that the sun is red, the sky is blue, and the cloud is white. She also expressed that the little lamb is hungry and looking for food. So, she gave the picture a name, 小 (xiăo) 羊 (yáng), which means little lamb, and wrote the two characters in the middle of the picture. The third picture shows a birthday card made by Kayla. Kayla first made five candles on the top, drew a unicorn, a Santa hat, and a Christmas tree on the left side of the card, and wrote characters, 五 (wǔ) 岁 (suì) 生 (shēng) 日(rì) which means five-year-old-birthday on the right side. Kayla showed her understanding of celebrating birthdays by using symbols such as drawings and writings which represent candles, cards, presents, and wishing. Kayla further told a simple story of how she interpreted the meaning of these symbols, and from there, she shared important moments of her everyday life experience.

\section{How Do Changes in Cultural and Social Environments Influence Children's Chinese Characters' Learning When They Are Temporarily Residing in the United States?}

\section{Increased Interactions with Technical Tools and Decreased Interactions with the Community}

Both families shared that children relied on Chinese learning apps, online videos, and books with other materials to learn Chinese characters when they were living in the United States because Chinese is less used in the community. For instance, Zara said,

Everyone speaks English and many of them also speak Spanish. Whenever we go, we see English and Spanish and there is no Chinese (Mandarin) at all. Even within Chinese community or meeting with Chinese friends, we also use English or combine both (English and Chinese Mandarin). So, it is hard to them (children) to have that (Chinese Mandarin characters) visualizations (Participant interview, 2020).

In other words, concept acquisition emerges from symbolic understanding and, thus is based in the culture and social world in which the child lives. "Culture and social environment are crucial elements that determine both the form of concept constitution and the acquisition of the concept" (Toran and Temel, 2012, p. 595). This, as seen in this study, becomes central to our research questions regarding children learning Chinese characters while temporarily residing in the United States. Vygotsky (1987) described everyday encounters and scientific direct instruction as the means by which children acquire conceptual understanding. Every day concepts include knowledge and learning that a child experiences in the course of living in a human body within a culture and society. Scientific concepts are 'adopted by the child in completed form from the domain of adult thinking' (Vygotsky, 1987, p. 169). These concepts are presented by a more knowledgeable other in the form of direct instruction or, perhaps, as an app or computer program.

In applying the two pathways of concept acquisition, it would seem that learning Chinese characters when not being situated in Chinese culture, would require that children take up the concepts associated with Chinese characters by way of scientific conceptual acquisition. This would imply direct instruction in the meaning associated with the abstract symbol, with the understanding of the symbol deconstructed back to the concrete referent and then back to the symbol. In contrast, children learning characters while immersed in Chinese culture would encounter characters as part of being embedded in the culture surrounded by environmental print. However, at the same time, children's everyday experiences with language provide a foundation for understanding the more abstract aspects of character learning. The mutual entanglement of the two ways of acquiring concepts allows for children's thinking to begin to move from simplistic to complex; from concrete to abstract and back to concrete; from the referent to the symbol and back to the referent. For children learning Chinese characters while residing in the United States, it was found that this change in cultural and social environment increased children's interaction with technical tools (scientific concept acquisition) and decreased their interactions in a Chinese community (every day concept acquisition). With learning being disproportionately presented through technical tools and scientific concept acquisition, it is unknown how this affects children's internalized understandings of the meaning of Chinese characters when this learning does not occur within Chinese cultural immersion. 


\section{Discussion}

This research study explored the development of a child's symbolic mind when learning Chinese characters, especially when Chinese children are temporarily residing in the United States. As the overall findings of the study analyzed how symbolic thought is developed and how symbols are used, it also sheds light on the relationship between symbolic representation and sociocultural differences as well as symbolic representation in non-alphabetic language learning. In this section, we took a deeper look from these two angles and provided our recommendations accordingly, one is we believed that all children have the capacity of symbolic representation but it is influenced by cultural differences, as well as development over time of cognitive capacities that encompass symbolic understanding, abstractions and language learning. Since development is time-dependent there are implications for instructional practice. Therefore, it is our suggestion when teaching young children Chinese characters that it is necessary to separate recognition and writing as two steps.

\section{Symbolic Representation is Influenced by Cultural Differences}

Based on conversations with the participants, it became understood that children perceive objects first, and then turn objects into pictorial production. This process is referred to as pictorial competencies by Rochat and Callaghan (2005, p. 3). According to Apperly, Williams, and Williams (2004), pictures are understood before written words, and the meaning discerned in pictures, as shown by the answers our participants provided, is always seen through a cultural lens. The impact of cultural influence and how children take that up play a crucial role in children's symbolic development and symbolic representation.

As the participants expressed, children were surrounded within a kind of sociocultural environment that impacts children's interactions with it. Wang (2018) states, "While adapting from one country to another and transforming from one culture to another, one's heritage language maintenance endures many changes-social, cultural, economic, and linguistic-along with changes to people's conceptions of life, societal values, and world outlook" (p. 253). For example, even children residing temporarily in the United States, tend to switch their language from Chinese to English to discover it and in turn, they gain more chances to acquire English rather than Chinese. In different sociocultural environments, an artifact, a decoration, an ornament, an item, a dish, a piece of clothing, a hairstyle, a toy, a gesture, and even eye contact is composed of cultural elements. Cultural influence is an invisible process that impacts children quietly. Rakoczy, Tomasello, and Striano (2005) state that "the symbolizing process always assumes a collective background of shared rules and practices for symbol making and interpreting" (p. 70). Indeed, children's understanding and development of symbolic actions with objects are in fact about cultural learning. It is through culture that shared understanding is defined. With natural observations and interactions, children imitate and sense cultural norms through various cultural (i.e. family) activities (Rakoczy et al., 2005).

Research participants further confirmed that they communicate with their children frequently regarding cultural differences between China and the United States. As they are currently living in the United States, there is a cultural comparison constantly occurring between the family's home culture and the culture in which they are temporarily residing. Where families make an effort to use Chinese language in the home, to provide exposure to Chinese media or artifacts, to share their cultural knowledge and understandings with their children, the children can experience, as well as comprehend, the differences (between Chinese and American culture) hand-in-hand and make connections with those differences to their language learning.

\section{Implications for Practice: Separating Recognition and Writing as Two Steps When Learning Chinese Characters}

Participants responded that to fully learn Chinese characters, that is, being able to recognize Chinese characters as well as write them, children should separate recognition and writing as two steps with recognition as the first step and followed by writing as the second step. As described earlier, the learning level, speed, and time can be set up through the Wukong Literacy app by parents who have a better 
understanding of their child/children's learning background. When the child is ready, such as having better fine motor skills and a foundation of character recognition, parents are able to introduce the character writing system to them. The app offers demonstration of character stroke order as well as the relevant practices, quizzes and games. Starting from here, on the one hand, participants stated that it is difficult to ask children to achieve visual recognition of characters, as well as writing at the same time. Recognition is usually easier compared to writing and it also occurs earlier in the developmental process than writing. Participants confirmed that they started to teach their children character recognition when their children were three years old, but children started to produce writing only after they turned four-years-old. It is understandable that children are still developing fine motor skills when they are three-years-old, their muscle control, balance, coordination between brain and action are not as mature as at four-years-old, thus, teaching children recognition first, then writing, makes sense.

On the other hand, Chinese characters consist of strokes and both strokes and characters must follow certain writing order rules. It is very hard for children to complete a character without following a correct stroke order, especially when a character has many strokes. At the same time, repetitive writing practice is commonly needed in memorizing the stroke order in reproducing characters. Repetitive movements (writing strokes) may establish a motor memory of the action, but for this to be internalized as having meaning, the motor movements should be accompanied by interaction with a 'more knowledgeable other' (Vygotsky, 1987) to situate the meaning linguistically within a cultural context. Hence, the participants emphasized that to achieve the ability to write is a slow process while children not only need to be mentallyphysically ready, but also should have enough mastery of word recognition. However, as children mature and can begin to produce and reproduce characters, the act of drawing and writing, as it involves the body in representing meaning, will ground and integrate previous character recognition in deeper symbolic understanding (Gibbs, 2006).

\section{Limitations of the study}

The limitations of this study are also recognized. For example, the study is limited to two families who temporally live in the United States; second, each family's perspective and experience brings stories that are specific to that family; and third, this study began in early 2020 and during the Covid-19 pandemic period with stay-at-home orders in place, which to some extent, reflects two families' strategies in assisting children's Chinese character learning and may be different from the way Chinese characters would be taught during regular times.

\section{Conclusion}

Symbolic representation and concept acquisition underlines much of children's language learning and development. Here this was studied through Chinese children's learning of Chinese characters in response to the research question of how do Chinese children learn to discern meaning from the symbols represented in Chinese characters? These two case studies offered some clues and they are, first, children become symbol-minded through interactions with more knowledgeable others, who through shared meaning and shared understanding of meaning, co-constitute a learners' 'culture' (Rakoczy et al., 2005). This introduction to meaning, whether through language learning or exposure in the environment, emerges through a cultural filter of shared meaning. When this shared meaning is intentionally introduced, as in the teaching of Chinese characters, learning and interacting in this way is cultural transmission of the underlying shared meaning of that culture, and the cultural mediator is language (Vygotsky, 1978). It can be said that cultural influence is infused in, not only the content of the language being learned, but also in the structure, the syntax, the grammar, and the phrasing of meaning (Boroditsky, 2001). Further, for a young child learning a language, the developing child's neural architecture forms in relation to information, cultural and otherwise, that is embedded in the process of learning that particular language. How a child understands symbolic information is greatly influenced by the child's exposure in the environment and to interaction with others (Fox, Levitt and Nelson, 2010).

In looking at this through the lens of relational developmental systems metatheory, a child, and thus, 
a child's development is holistic and occurs simultaneously in a mutually-reinforcing interaction between mind and body "that extends mental processes out into the body and into the technological and cultural worlds" (Overton, 2013, p. 36). Thus, under this theoretical construct, cultures, individuals and communities are "co-constructed, co-determined, and co-developed" (Overton, 2013, p. 36). It would seem that the children in this study who are learning Chinese characters when not immersed only in Chinese culture, are being exposed to Chinese cultural components intentionally by their parents. Since the exposure to cultural components is not spontaneous, as it would be if they lived in China, then their first uptake of the cultural aspects of the symbols is coming by way of what Vygotsky (1987) called scientific conceptual acquisition. However, since these children are also simultaneously learning other languages (English, Spanish) and the associated symbols (alphabet), prior learning from spontaneous concept acquisition is present and transferrable to support their scientific conceptual acquisition of Chinese Mandarin characters.

The second clue to how Chinese children discern meaning from Chinese characters can be seen when utilizing digital aids such as apps to demonstrate the evolution of Chinese characters. The animation process seems to help children engage in every day (spontaneous), as well as scientific, concept acquisition (Fleer and Raban, 2006; Vygotsky, 1987) by way of the process described by Vygotsky. Further, in encouraging children's drawings to reinforce symbolic representation, connections are established between children's production of images and the application of the appropriate Chinese character/s. The last clue can be seen in the participants' suggestion that character recognition and character writing be separated into two steps, as these two items require different skill sets and levels of development. Being able to recreate a Chinese character may indicate an understanding of the symbol or it may indicate high levels of fine motor skills and imitation. Writing Chinese characters may not give as much information about symbolic representation as the ability to recognize characters. However, once children begin to recognize the characters, the meaning can be internalized through the (embodied) motor process of (re)producing the symbols through writing (Yeh et al., 2017). While it is acknowledged that use of apps, such as Wukong Literacy, are helpful because they are designed to demonstrate character evolution, capture children's attention and provide structure for learning practice, it is also understood that due to the cultural basis of language and meaning, children still need social interaction during the learning process to fully comprehend the symbolism inherent in language and specifically, Chinese characters.

It appears that two, mutually reinforcing modes of acquiring understanding about the world, i.e. the process of constructing concrete mental models into abstractions and deconstructing from an abstract concept to a concrete item, are required for children to develop symbolic thought and abstract concepts. The recognition of Chinese characters, and the later step of symbolic representation through writing, exemplifies this relational developmental process where meaning is emergent through the interaction of spontaneous exposure to culture and directed instruction.

\section{Declarations}

Acknowledgements: We acknowledge our participants' engagement in our research study. We appreciate their generous time and contribution. We also want to thank the company of Wukong Literacy which gave us permission to use their copyrighted material. Many thanks to the New Mexico State University College of Education's School of Teacher Preparation, Administration, and Leadership, as well as the New Mexico State University Office of Research Integrity and Compliance for support of this, and other, early childhood research.

Authors' contributions: The authors of this paper have equal contribution.

Competing interests: The authors declare that they have no competing interests.

Funding: The authors declare that there were no funding sources for this research.

\section{References}

Abbott, C. (2000). Symbols now. Leamington Spa: Widgit Software.

Apperly, I. A., Williams, E., \& Williams, J. (2004). Three-to four-year-olds' recognition that symbols have a stable meaning: Pictures are understood before written words. Child Development, 75(5), 1510-1522. https://doi.org/10.1111/j.1467-8624.2004.00754.x 
Learning Chinese Mandarin characters in an English-speaking...

Athey, C. (2007). Extending thought in young children: A parent-teacher partnership (2 ${ }^{\text {nd }}$ ed.). London: Paul Chapman Publishing. https://doi.org/10.4135/9781446279618

Black Delfin, A. (2020). The discursive and the material in early childhood play: Co-constitution of gender in open and subversive spaces. Gender and Education, 1-16. https://doi.org/10.1080/09540253.2020.1786012

Boroditsky, L. (2001). Does language shape thought?: Mandarin and English speakers' conceptions of time. Cognitive Psychology, 43(1), 1-22. https://doi.org/10.1006/cogp.2001.0748

Bruner, J. S. (1966). Towards a theory of instruction. New York: Norton.

Creswell, J. W., \& Plano Clark, V. L. (2011). Designing and conducting mixed methods research (2nd ed.). Los Angeles, CA: Sage Publications.

DeLoache, J. S. (2004). Becoming symbol-minded. Trends in Cognitive Sciences, 8(2), 66-70. https://doi.org/10.1016/j.tics.2003.12.004

Elkins, J. (2003). Visual studies: A skeptical introduction. New York, NY: Routledge.

Fadjo, C., Lu, M., \& Black, J. (2009). Instructional embodiment and video game programming in an after school program. In Proceedings of World Conference on Educational Multimedia, Hypermedia and Telecommunications (pp. 4041-4046). Chesapeake, VA: AACE.

Fleer, M., \& Raban, B. (2006). A cultural-historical analysis of concept formation in early education settings: Conceptual consciousness for the child or only the adult?. European Early Childhood Education Research Journal, 14(2), 69-80. https://doi.org/10.1080/13502930285209921

Fox, S. E., Levitt, P., \& Nelson, C. A. 3rd. (2010). How the timing and quality of early experiences influence the development of brain architecture. Child Development, 81(1), 28-40. https://doi.org/10.1111/j.1467-8624.2009.01380.x

Gayman, A. M., Fraser-Thomas, J., \& Baker, J. (2017). Relational developmental systems metatheory: A conceptual framework to understand and promote older adults' involvement in sport. European Review of Aging and Physical Activity, 14(1), 1-10. https://doi.org/10.1186/s11556-017-0182-6

Gee, J. P. (1990). Social linguistics and literacies: Ideology in discourses, critical perspectives on literacy and education. London: Routledge.

Gibbs, R. W. (2006). Embodiment and cognitive science. Cambridge: Cambridge University Press.

Glenberg, A. M. (2008). Toward integration of bodily states, language, and action. In. Semin, G. R. \& Smith, E. R. (Eds.), Embodied grounding: Social, cognitive, affective, and neuroscientific approaches (pp. 43-70). New York: Cambridge Univ. Press. https://doi.org/10.1017/CBO9780511805837.003

Hammond, J. (Ed.). (2002). Scaffolding teaching and learning in language and literacy education. Newtown: PETA.

Inhelder, B., \& Piaget, J. (1964). The early growth of logic in the child. London: Routledge and Kegan Paul.

Lu, M. T., Wu, C. Y., Fadjo, C., \& Black, J. (2010, March). Future trends in Chinese character teaching: Use of embodiment and technologies in classrooms. In Society for Information Technology \& Teacher Education International Conference (pp. 2485-2492). Association for the Advancement of Computing in Education (AACE).

McBride, C. A. (2016). Is Chinese special? Four aspects of Chinese literacy acquisition that might distinguish learning Chinese from learning alphabetic orthographies. Educational Psychology Review, 28(3), 523-549. https://doi.org/10.1007/s10648-015-9318-2

Menezes, V. (2013). Second language acquisition: Reconciling theories. Open Journal of Applied Linguistics, 3(7), 404-412. https://doi.org/10.4236/ojapps.2013.37050

Mercer, J. (2018). Child development: Concepts and theories. London: Sage.

Namy, L. L., \& Waxman, S. R. (2005). Afterword: Symbols redefined: Developmental and comparative perspectives. In L. L. Namy (Ed.), Symbol use and symbolic representation: Developmental and comparative perspectives. Mahwah, NJ: Lawrence Erlbaum Associates Publishers.

Otsuka, K., \& Jay, T. (2017). Understanding and supporting block play: Video observation research on preschoolers' block play to identify features associated with the development of abstract thinking. Early Child Development and Care, 187(5-6), -990-1003. https://doi.org/10.1080/03004430.2016.1234466

Overton, W. (2013). Relationism and relational developmental systems: A paradigm for developmental science in the post-cartesian era. In R. Lerner, \& J. B. Benson (Eds.), Advances in child development and behavior (Vol. 44, pp. 21-57). Elsevier. https://doi.org/10.1016/B978-0-12-397947-6.00002-7

Pampoulou, E., \& Detheridge, C. (2007). The role of symbols in the mainstream to access literacy. Journal of Assistive Technologies, 1(1), 15-21. https://doi.org/10.1108/17549450200700004

Patton, M. Q. (2002). Qualitative research and evaluation methods. Thousand Oaks, CA: Sage Publications.

Piaget, J. (1950). The psychology of intelligences. London: Routledge \& Kegan Paul. 
Wenjie WANG \& Annabelle BLACK DELFIN

Piaget, J., \& Inhelder, B. (1969). The psychology of the child. New York: Basic Books.

Rakoczy, H., Tomasello, M., \& Striano, T. (2005). How children turn objects into symbols: A cultural learning account. In L. L. Namy (Ed.), Symbol use and symbolic representation: Developmental and comparative perspectives (pp. 69-97). Mahwah, NJ: Lawrence Erlbaum Associates Publishers.

Rochat, P., \& Callaghan, T. (2005). What drives symbolic development? The case of pictorial comprehension and production. In L. L. Namy (Ed.), Symbol use and symbolic representation: Developmental and comparative perspectives (pp. 25-46). Mahwah, NJ: Lawrence Erlbaum Associates Publishers.

Saldaña, J. (2009). The coding manual for qualitative researchers. Thousand Oaks, CA: Sage Publications Ltd.

Smith, L. B., \& Jones, S. S. (2011). Symbolic play connects to language through visual object recognition. Developmental Science, 14(5), 1142-1149. https://doi.org/10.1111/j.1467-7687.2011.01065.x

Sun, L. (2015). Radical: A learning system for Chinese Mandarin characters. Retrieved from https://repository.library.northeastern.edu/files/neu:rx915j987/fulltext.pdf

Taylor, K. B. (2016). Diverse and critical perspectives on cognitive development theory. New Directions for Student Services, 154, 29-41. https://doi.org/10.1002/ss.20173

Thompson, C. M. (1995). The visual arts and early childhood learning. Reston, Va.: National Art Education Association.

Tong, X., Tong, X., \& McBride, C. (2017). Radical sensitivity is the key to understanding Chinese character acquisition in children. Reading and Writing, 30(6), 1251-1265. https://doi.org/10.1007/s11145-017-9722-8

Toran, M., \& Temel, Z. F. (2012). An examination of Turkish preschool curriculum's effect on children's concept acquisition. ProcediaSocial and Behavioral Sciences, 47, 594-599. https://doi.org/10.1016/i.sbspro.2012.06.701

Uttal, D. H., \& Yuan, L. (2014). Using symbols: Developmental perspectives. Wiley Interdisciplinary Reviews: Cognitive Science, 5(3), 295304. https://doi.org/10.1002/wcs. 1280

Vallotton, C. D., \& Ayoub, C. C. (2010). Symbols Build Communication and Thought: The role of gestures and words in the development of engagement skills and social-emotional concepts during toddlerhood. Social Development, 19(3), 601-626. https://doi.org/10.1111/j.1467-9507.2009.00549.x

van Oers, B. (2001). Contextualisation for abstraction. Cognitive Science Quarterly, 1(3), 279-305.

van Oers, B., \& Poland, M. (2007). Schematising activities as a means for encouraging young children to think abstractly. Mathematics Education Research Journal, 19(2), 10-22. https://doi.org/10.1007/BF03217453

Veraksa, A., \& Veraksa, N. (2016). Symbolic representation in early years learning: The acquisition of complex notions. European Early Childhood Education Research Journal, 24(5), 668-683. https://doi.org/10.1080/1350293X.2015.1035539

Vygotsky, L. S. (1978). Mind in Society: The development of higher psychological processes. In. M. Cole, V. John-Steiner, S. Scribner, \& E. Souberman (Eds.), Cambridge, MA: Harvard University Press.

Vygotsky, L. S. (1981). The genesis of higher mental functions. In J. V. Wertsch (Ed.), The concept of activity in Soviet psychology (pp. 144-188). Armonk, NY: Sharpe.

Vygotsky, L. S. (1987). Thinking and speech. In R. W. Rieber and A. S. Carton (Eds.), The collected works of L.S Vygotsky, Vol.1: Problems of general psychology (p. 39-285). New York: Plenum Press.

Wang, W. (2018). Cases of heritage language maintenance among Asian immigrant families in the southwestern United States (Doctoral Dissertation). New Mexico State University, New Mexico [Publication No. 13426314].

Wu, J. (2016). The origins of 670 essential words. Boston: Cheng \& Tsui Company, Inc.

Wu, L. Y. (2013). Children's play and symbolic representation. Review of Global Management and Service Science, 3, 7-14.

Wukong Literacy. (2020). Characters learning. Retrieved November 27, 2020, from https://www.gongfubb.com/

Yeh, S. L., Chou, W. L., \& Ho, P. (2017). Lexical processing of Chinese sub-character components: Semantic activation of phonetic radicals as revealed by the Stroop effect. Scientific Reports, 7(1), 1-12. https://doi.org/10.1038/s41598-017-15536-w

\section{Appendices}

\section{Appendix A Parent Questionnaire}

Please fill out this questionnaire to the best of your ability. There are no right or wrong answers, and the intent to this questionnaire is to help us get to know your child/children's Chinese learning. 
Basic information

1. Child/children's name

2. Child/children's age

3. Child/children's gender

4. Number of siblings

5. You and your child/children's length of stay in the U.S.

Language learning

\begin{tabular}{|l|l|l|}
\hline \multicolumn{1}{|c|}{ Question/country } & China & United States \\
\hline 6. Child/children home language(s) & & \\
\hline 7. Child/children school language(s) & & \\
\hline $\begin{array}{l}\text { Length of time learning Chinese (speaking, listening, reading, } \\
\text { and writing) }\end{array}$ & & \\
\hline 9. Place(s) of learning Chinese & & \\
\hline 10. Way(s) of learning Chinese & & \\
\hline Your observation and reflection & & \\
\hline 11. Child/children's learning result & & \\
\hline 12. Child/children's interests & & \\
\hline The learning differences between the two countries & & \\
\hline 14. The challenges of learning Chinese & & \\
\hline 15. Other thoughts & & \\
\hline
\end{tabular}

\section{Appendix B Parent Interview}

1. How many language(s) is/are your child/children learning now? And at what age did you start teaching your child/children those languages?

2. What is your child/children's Chinese level? Can you explain it from the skills of listening, speaking, reading, and writing?

3. How does your child/children learn to recognize Chinese characters? Do you have specific methods?

4. How does your child/children learn to write characters? Can you share some strategies?

5. How does your child/children learn Chinese in China? Can you provide some examples?

6. How does your child/children learn Chinese in the United States? Can you provide some examples?

7. What are the differences between learning Chinese characters in China and the United States?

8. What challenges do you have when you help your child/children learn Chinese characters when you are in the United States? And how do you handle those challenges?

9. What suggestions do you want to give to families, parents, children, and educators who are also engaged in Chinese learning?

10. Do you have any other thoughts that you would like to share? 